\title{
Arbor
}

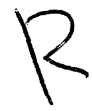

\section{Seguridad y riesgo de toxicidad de los alimentos: un debate actual}

\author{
Abel Mariné y M. Carmen Vidal
}

Arbor CLXVIII, 661 (Enero 2001), 43-63 pp.

El progreso científico y tecnológico ha tenido muchas consecuencias beneficiosas para la humanidad pero también conlleva riesgos. Un ámbito en el que estos dos aspectos de la cuestión se manifiestan más claramente es el de la alimentación humana. Las posibilidades actuales de la industria alimentaria permiten la obtención de productos cada vez más adaptados a las necesidades y gustos del consumidor. No obstante, existe una desconfianza frente a los alimentos transformados que se juzgan, injustamente, como menos seguros que los frescos o naturales. Ciertos escándalos de gran resonancia alimentan esta desconfianza, y no suele tenerse en cuenta que las vacas locas, los pollos con dioxinas o la carne con hormonas, por ejemplo, son problemas de las fases primarias de producción, agrícola y ganadera, y no de la industria alimentaria propiamente dicha. Bajo la perspectiva global de lo que son los alimentos y de lo que conocemos de su composición $y$ funciones en el ser humano, $y$ teniendo en cuenta de que el riesgo cero no existe para ninguna actividad, y por lo tanto tampoco en la alimentación, se puede afirmar que nuestros alimentos nunca han sido tan seguros como en la actualidad, aunque sigue habiendo problemas $y$ en un tema como éste, de evidente implicación sanitaria, toda precaución es poca.

En este artículo se discuten las relaciones entre alimentación y riesgo y la percepción social sobre estas relaciones, se describen someramente los componentes tóxicos naturales de los alimentos, la contaminación de los mismos, los problemas de alteración y deterioro, así como la adulteración y el fraude y sus consecuencias. También se aborda la significación de casos actuales que cuestionan la seguridad de los alimentos y el papel del control alimentario, por parte de productores $y$ 
administraciones públicas, para garantizar la calidad y la seguridad de los alimentos.

\section{Introducción}

El «Comité de Protección de los Alimentos» de los Estados Unidos definió en 1959 la seguridad de los alimentos como «la práctica certeza de que no se derivará ningún daño o lesión del uso de una substancia en una forma o cantidad propuesta». Esto significa que cabe esperar y exigir que se pueda alcanzar una razonable seguridad de que los alimentos que ingerimos, y por lo tanto las substancias naturales o propias así como las añadidas que contienen, no causarán ningún efecto negativo sobre el organismo. Bajo esta perspectiva estamos considerando los mínimos que debe cumplir un alimento, es decir, la ausencia de cualquier forma de adulteración, deterioro o contaminación que pueda dar lugar a efectos tóxicos o indeseables para el consumidor. Aunque sea obvio, hay que recordar y destacar que los objetivos de la ingestión de alimentos van mucho más allá, dado que aportan nutrientes esenciales para el mantenimiento de todas las funciones vitales, componentes con efectos beneficiosos a medio y largo plazo, placer en su consumo (el valor gastronómico es importante) e incluso satisfacen deseos de prestigio o status social.

En el mundo en que vivimos, con un desarrollo tecnológico acelerado que rápidamente se transforma en productos (entre ellos nuevos alimentos) y servicios (nuevas tecnologías de tratamiento industrial y culinario de los mismos) al alcance de la población con suficiente poder adquisitivo, subsiste permanentemente una desconfianza con respecto a los alimentos, especialmente los industrializados. Ciertos hechos parecen confirmar esta desconfianza, cuando en realidad se trata de las excepciones, no de la regla.

\section{Alimentación y riesgo}

Teniendo en cuenta los datos a partir de los cuales se considera un producto como seguro, hay que admitir que estimar el nivel inocuo de un contaminante de un alimento, por ejemplo, siempre tendrá un inevitable margen de incertidumbre. Nunca se insistirá bastante en destacar que esto también ocurre en cualquier otra actividad humana, y baste citar, por obvio, el riesgo que supone circular en coche, sobre 
todo en un fin de semana. En la mayoría de los casos, a lo más que podemos aspirar es a definir de la manera más clara posible el eventual riesgo asociado a unas particulares condiciones de exposición a una sustancia determinada.

Si consideramos la cuestión del riesgo respecto a la contaminación de los alimentos, hay que recordar que el alimento no contaminado nunca ha existido. Es más, si tenemos en cuenta todas las formas posibles de contaminación, nuestra dieta actual es la menos contaminada de la historia humana. Es un hecho evidente que la esperanza de vida ha aumentado de forma espectacular en las sociedades desarrolladas desde principios del siglo XX hasta hoy en día, y es indudable que la alimentación ha sido un elemento importante de este progreso.

Aunque se trate de una referencia literaria, citemos como ejemplo ilustrativo de una situación anterior una descripción que hace Upton Sinclair, en su gran novela «La jungla» publicada en Estados Unidos en 1906: "Había reses que, cebadas con la malta de los desperdicios de las fábricas de cerveza, tenían todo el cuerpo cubierto de tumores. $\mathrm{Y}$ era un trabajo verdaderamente asqueroso y repugnante matar estos animales, puesto que, al hundir el cuchillo en el cuerpo de la res, los tumores se reventaban y salpicaba por todas partes el pus hediondo y verdaderamente intolerable...Pues con este material se fabricaba la llamada 'carne embalsamada', que ocasionó más víctimas entre los soldados americanos durante la guerra de Cuba que las balas de los españoles». Esto hoy ya no ocurre, entre otras razones porque la publicación de esta obra fue un elemento determinante para que Estados Unidos promulgara, como ya habían hecho diversos países europeos, normas legislativas sobre la seguridad y control de los alimentos y sus componentes. Otro salto cualitativo importante en la evaluación de la seguridad de los productos destinados al consumo humano se dio en la década de 1960, a raíz de la observación de los efectos teratógenos del fármaco talidomida. El rigor, con criterios internacionales, que se introdujo a partir de este momento en la evaluación de aditivos alimentarios, ingredientes y en nuevos alimentos es la mejor garantía de que si los estudios (toxicológicos, nutricionales y de equivalencia de los nuevos alimentos con respecto a los tradicionales aceptados como seguros) se llevan a cabo correctamente la evaluación riesgo/beneficio aporta toda la seguridad posible, en el bien entendido que debe asumirse que el riesgo cero no existe en ninguna actividad humana. Recordemos que los ensayos toxicológicos que se realizan incluyen estudios toxocinéticos, de genotoxicidad, de alergenicidad potencial, de capacidad de colonización del tracto gastrointestinal por 
parte de los microorganismos que contienen los alimentos, de patogenicidad, ensayos de efectos subcrónicos, estudios sobre efectos en la reproducción y de carcinogenicidad.

\section{Percepción social del riesgo alimentario}

Como ya ponía de manifiesto Golberg en 1971, el clima de opinión frente a los alimentos, especialmente los elaborados por la industria, plantea el problema de si es aceptable para el consumidor estar expuesto a un riesgo potencial, aunque remoto, que debe ser compartido, de hecho inconscientemente, por un vasto número de personas de edades y estados de salud diversos. Se considera por parte de los consumidores que este nivel de seguridad no basta, ya que en definitiva se estiman como inadecuados, o insuficientes, los argumentos basados en que el uso más o menos prolongado de una sustancia, por ejemplo un aditivo alimentario, sin que se hayan observado efectos negativos confiere un grado suficiente de garantía. A este respecto es muy ilustrativo el caso del aditivo alimentario edulcorante sacarina. Su descubrimiento y uso son anteriores a los estrictos - y lógicos - criterios actuales de autorización de un nuevo aditivo o ingrediente alimentario. Cuando, hace ya algunos años, las autoridades sanitarias de los Estados Unidos se plantearon su prohibición, las asociaciones de diabéticos, usuarios tradicionales de sacarina, se opusieron a esta medida, que les privaría de tener acceso a muchas formas de productos dulces. De hecho la sacarina puede ser cancerígena en ciertos animales de laboratorio y en determinadas condiciones experimentales, pero parece suficientemente demostrado que no lo es en las dosis usadas como ingrediente de productos edulcorados artificialmente. En este caso los consumidores, conocedores de estos hechos, asumieron conscientemente y libremente «su riesgo», porque ya conocían y valoraban por experiencia propia los beneficios. El problema surge, sobre todo, cuando el consumidor cree que no conoce o no controla lo que estima como riesgos y peligros y/o cuando no conoce o valora los beneficios de la incorporación de un aditivo, por ejemplo.

Por otro lado, las opiniones de los consumidores han evolucionado en estas últimas décadas, tal como se demuestra en las encuestas que hizo el Food Market Institute de los Estados Unidos, sobre los temas que preocupaban a los consumidores con respecto a los alimentos, en 1983 y en 1995. En 1983 la primera inquietud eran los aditivos alimentarios (injustamente considerados como contaminantes de hecho 
o como indicadores de manipulación incorrecta por parte de la industria alimentaria), y en 1995 las primeras preocupaciones eran el contenido en grasas, sal o colesterol. Aunque con un cierto retraso, lo mismo ha ocurrido en Europa; es decir, actualmente preocupa más la relación alimentación/salud respecto a la naturaleza de los alimentos que consumimos que su posible falta de seguridad, que en principio se considera suficiente. No obstante, esta situación se trastoca cuando se producen accidentes que dan lugar a intoxicaciones reales o a riesgos percibidos como graves, como es el caso de las llamadas «vacas locas» o las dioxinas en los pollos belgas.

Es muy ilustrativo de la percepción contraria de los consumidores a los que consideran «alimentos industrializados» el que, según un reciente sondeo llevado a cabo por el Institut Català de Consum, no se han cambiado los hábitos a raíz de estos escándalos alimentarios porque siguen suscitando más confianza los productos agrícolas que los procesados por la industria alimentaria, a pesar de que dichos escándalos están más vinculados al sector primario que al industrial.

\section{Componentes tóxicos naturales de los alimentos}

Una idea muy arraigada entre los que no son expertos en los alimentos es que los productos naturales son inocuos y que el peligro siempre procede de los productos transformados. Ello no es necesariamente así. En efecto, incluso un alimento natural en buen estado y no manipulado o manipulado de forma correcta puede provocar efectos adversos por causas diversas (entre las que no es la única la sensibilidad individual), dado que el organismo humano no distingue entre natural o trasformado o sintético, sino entre perjudicial o inocuo.

Debemos tener en cuenta que los componentes (macrocomponentes y microcomponentes) naturales o propios de un alimento son muchos y muy diversos (en general se pueden contar por centenares). De hecho, si muchos de estos compuestos se sometieran a los actuales ensayos de seguridad o inocuidad, resultarían tóxicos y no podrían incorporarse en ningún caso a los alimentos. Cabe citar como ejemplo a la cafeína por su actividad mutagénica in vitro; si hoy se presentara como una sustancia «nueva» para ser autorizado su consumo en alimentación humana probablemente no pasaría las actuales barreras legales. La suerte de la cafeína, si podemos expresarnos así, es que viene siendo consumida desde hace siglos, y aunque sus acciones estimulantes del sistema nervioso central hacen que su consumo deba ser moderado, 
nada indica que su ingestión en cantidades razonables revista ningún peligro. Análogos razonamientos podríamos hacer, y con mayor motivo, respecto al alcohol etílico.

Sin pretender ser exhaustivos podemos citar una serie de ejemplos de toxicidad natural de los alimentos, ya sea porque contienen en origen substancias potencialmente tóxicas o porque éstas se generan durante los tratamientos de obtención de alimentos o en los procesos culinarios tradicionales. Igualmente, pueden presentarse efectos indeseables y/o tóxicos por el consumo de alimentos naturales por parte de individuos con situaciones metabólicas o farmacológicas particulares.

Las toxinas paralizantes, diarreicas, amnésicas y neurotóxicas de los moluscos se forman debido a complejos ciclos meteorológicos y biológicos del medio ambiente marino, en los que no parece intervenir la actividad humana. Estos tóxicos son producidos fundamentalmente por dinoflagelados que los moluscos filtran. Por causas parecidas se pueden encontrar peces comestibles que son tóxicos si se consumen en determinadas épocas (ictiotoxismo).

Las micotoxinas son substancias tóxicas producidas por hongos microscópicos del medio ambiente que afectan a los animales y al ser humano. Contaminan a los alimentos en origen o a causa de un acondicionamiento deficiente. Se pueden encontrar en alimentos como frutos secos o cereales. Entre ellas podemos destacar las aflatoxinas que son poderosos tóxicos hepáticos y cancerígenos.

Muchos metales como selenio, mercurio, plomo, cromo, arsénico y otros, pueden encontrarse en elevadas concentraciones, y por tanto tóxicas, en alimentos, como consecuencia de las características geológicas de los terrenos en que se cultivaron los vegetales.

Las alergias de origen alimentario, no sólo son debidas a proteínas sino incluso a moléculas de menor tamaño. Pueden ser provocadas por alimentos naturales y en buen estado como cacahuetes, fresas, leche, huevos, trigo, marisco, apio, chocolate y otros. En realidad son mucho más frecuentes las alergias debidas a componentes naturales de los alimentos que las debidas a ciertos aditivos alimentarios.

Hay intolerancias alimentarias, de origen no alérgico, como la intolerancia a la lactosa, que hace a los afectados intolerantes a la leche. Este es un trastorno muy extendido que aparece casi siempre después del normal periodo de lactancia. De hecho una tolerancia generalizada a la lactosa en adultos sólo se da en los pueblos de origen nórdico y en algunas tribus pastoriles africanas, todos ellos habituales $\mathrm{y}$ ancestrales consumidores de leche. 
Una forma de manifestación tóxica de los alimentos, que puede parecer singular pero que no es insólita, la constituyen las interacciones entre alimentos y medicamentos, debidas a alimentos en buen estado y fármacos en principio prescritos y dosificados correctamente. Citemos como ejemplo el denominado "sindrome del queso", que consiste en unas crisis hipertensivas (a veces mortales) desencadenadas al ingerir ciertos quesos $u$ otros alimentos ricos en aminas biógenas por parte de individuos sometidos a tratamientos con medicamentos inhibidores de la mono-amino-oxidasa (IMAO).

El favismo, es un síndrome hemolítico debido a la ingestión de habas, causado por un defecto genético, que se da sobre todo en el área mediterránea y en el que hay riesgo de muerte.

Los errores innatos del metabolismo, como la fenilcetonuria o la galactosemia, debidos a defectos genéticos, impiden metabolizar la fenilalanina (aminoácido esencial) y la galactosa (componente de la lactosa de la leche) respectivamente. Conllevan graves trastornos metabólicos, para los que la dietética es fundamental para prevenir los efectos y para el tratamiento.

Algunos desequilibrios en la dieta pueden llegar a causar efectos tóxicos cuando diversos alimentos, en perfecto estado e incluso recomendables en cantidades normales, se consumen en exceso dentro de dietas monótonas (que nunca son aconsejables). Citemos por ejemplo diversos tipos de plantas crucíferas (coles y similares), que pueden llegar a ser bociógenas (generadoras de bocio), ciertos pescados de agua dulce que contienen antagonistas de la vitamina B1, o leguminosas que contienen inhibidores de enzimas digestivos que pueden llegar a causar retraso en el crecimiento e hipertrofia pancreática. Hay que señalar también que algunos de los componentes causantes de estos efectos se destruyen total o por lo menos parcialmente con la cocción o los tratamientos térmicos. Es decir, en este caso la tecnología industrial o culinaria aportan la solución.

Los tratamientos tecnológicos o culinarios inadecuados pueden dar lugar, por ejemplo, a grasas sobrecalentadas, en las que se pueden formar compuestos tóxicos como peróxidos o hidrocarburos policíclicos. Por otro lado una cocción insuficiente de algunas leguminosas determinará que no se destruyan las fitohemaglutininas tóxicas que contienen.

Hay substancias naturales cancerígenas, como algunos derivados del selenio que se encuentran en cereales cultivados en terrenos seleníferos, o los hidrocarburos policíclicos que se encuentran en alimentos ahumados con humo natural o las aminas heterocíclicas que 
se generan al calentar a temperaturas elevadas (asados, grill, parrilla, brasa) alimentos ricos en proteínas como carne y pescado.

\section{Contaminación biótica de los alimentos}

La contaminación biótica de los alimentos se debe a la presencia no deseada de microorganismos, virus, parásitos y/o productos tóxicos de origen biológico.

Es importante destacar que este tipo de contaminación está mucho más asumida por el consumidor que la abiótica, lo cual seguramente se debe a que es más conocida, en sus causas y efectos. Así, una intoxicación alimentaria causada por una salsa mayonesa u otra salsa casera (o en restauración colectiva) no desencadena una reacción de alarma tan grande como cualquier contaminación que es o se considera de origen industrial. Este hecho no deja de resultar paradójico, si tenemos en cuenta que el número de casos de este tipo es muy superior a los que se deben a contaminaciones abióticas, aún teniendo en cuenta que el registro y control de las abióticas está mucho menos desarrollado y es de más difícil seguimiento, en especial si se consideran, y así debe ser, los eventuales efectos crónicos.

Como señalan Herrera y Conchello (1999), las enfermedades de origen microbiano y de transmisión alimentaria son en la actualidad de índole universal, tanto desde el punto de vista de la salud pública como desde el económico. En España el número de casos de toxiinfecciones alimentarias registradas es de aproximadamente 12.000 al año (con una media de aproximadamente 1000 brotes/año). Teniendo en cuenta que el número de casos declarados se estima que es entre el 1 y el 10 por ciento de los que realmente ocurren, resulta evidente que la frecuencia de estas intoxicaciones es muy elevada y su coste sin lugar a dudas excesivo.

Los microorganismos más implicados son las Salmonella, aunque se registra un crecimiento de las gastroenteritis producidas por Campylobacter. También debemos citar Staphylococcus aureus, Clostridium perfringens, Clostridium botulinum, Yersinia enterocolitica y Escherichia coli (entérico). La contaminación por estos microorganismos puede tener lugar en la granja, en los procesos de transformación o bien durante la preparación de los alimentos en el hogar o en los establecimientos de restauración. Deben destacarse por su frecuencia las contaminaciones no vinculadas con la producción propiamente dicha, es decir en el hogar o en los establecimientos de restauración, a pesar de lo cual 
los productores siempre suelen ser los que se llevan la «mala prensa». Como también señalan Herrera y Conchello, las causas primordiales que explican la alta incidencia de este tipo de intoxicaciones y/o toxiinfecciones son el rápido incremento de la población mundial, el crecimiento expansivo de la residencia urbana, la modificación de los sistemas de oferta alimentaria, la aparición de nuevos hábitos alimentarios (restauración colectiva, restauración diferida, preparación de comidas precocinadas, etc.), los avances importantes en tecnología alimentaria no asimilados totalmente por el colectivo de consumidores, y el incremento desmesurado de los movimientos poblacionales (turismo, refugiados, emigración, etc.). Todo ello ha propiciado la aparición de las llamadas «enfermedades emergentes» de origen alimentario, entendiendo por tales las que han aparecido recientemente en una población, o que existían antes pero cuya extensión ha aumentado rápidamente, o incluso enfermedades no descritas anteriormente.

\section{Contaminación abiótica de los alimentos}

La contaminación abiótica se debe a la presencia en los alimentos de productos químicos o residuos de los mismos. La OMS define como residuo cualquier sustancia que persiste en un medio tras haber sido introducido en él, voluntariamente o no, y cuya presencia es cualitativa o cuantitativamente anormal. También hay que considerar los contaminantes de naturaleza radioactiva.

Las fuentes de contaminación de origen industrial pueden ser los vertidos de industrias y los residuos procedentes de equipos y maquinaria o de envases y embalajes. También algunos procesos tecnológicos pueden dar lugar a formación de contaminantes químicos, como las nitrosaminas o los hidrocarburos aromáticos policíclicos resultantes del ahumado. Las substancias que más frecuentemente son responsables de la contaminación abiótica de los alimentos son: metales pesados, hidrocarburos policíclicos, aminas heterocíclicas y plaguicidas. Indiquemos por su singularidad el "plumbismo" o saturnismo que se está dando en España en aves silvestres por la ingestión del plomo de los perdigones que estas aves encuentran en el medio en el que se desenvuelven, y que puede afectar al consumidor que cree ingerir animales en su estado natural y por lo tanto sin riesgo de productos extraños en su carne. En el caso de los pesticidas o plaguicidas conviene recordar que su presencia en alimentos no sólo se debe a tratamientos fitosanitarios inadecuados sino también a la impregnación ambiental de 
los mismos, problema que puso dramáticamente en evidencia Rachel Carson al publicar en 1962 su libro Silent Spring (Primavera silenciosa).

A efectos de valorar la toxicidad de los alimentos contaminados es muy importante disponer de datos cuantitativos sobre las concentraciones de estos contaminantes.

Las nitrosaminas constituyen un ejemplo muy ilustrativo de las opciones que hay que tomar ante un problema de seguridad que presenta diversos enfoques, y que exponemos muy sintéticamente. En la elaboración de derivados cárnicos curados (embutidos) se utilizan nitratos y nitritos, que tienen un efecto estabilizador del color y protector frente a la contaminación por Clostridium botulinum, responsable de una grave intoxicación alimentaria, que se evita al utilizar estos aditivos. No obstante, los nitritos (añadidos o procedentes de nitratos) pueden reaccionar con aminoácidos y aminas, que se encuentran de forma natural en estos productos, y formar nitrosaminas potencialmente cancerígenas. Evitar este segundo riesgo, es decir prescindir de estos aditivos, comportaría aumentar el de botulismo. Por esta razón, el uso de nitratos y nitritos está reglamentado y debe hacerse en condiciones muy controladas, para reducir al mínimo posible el contenido en nitrosaminas. Se han ensayado tecnologías que permitirían prescindir totalmente de nitratos y nitritos pero el costo de producción es mayor. De una forma muy esquemática el riesgo cero en este caso sería no consumir embutidos curados con sales nitrificantes y, aún cuando ningún alimento es imprescindible dentro de una dieta variada, no parece que sea conveniente ni que un consumo moderado de los mismos, bien elaborados, aumente el riesgo de cáncer.

\section{Alteración y deterioro de los alimentos}

Según el Código Alimentario Español, alimento alterado es todo alimento que durante su obtención, preparación, manipulación, transporte, almacenamiento o tenencia, y por causas no provocadas deliberadamente, sufre variaciones en sus caracteres organolépticos o sensoriales (color, aroma, textura, sabor), composición química o valor nutritivo, de tal forma que su aptitud para el consumo queda anulada o sensiblemente disminuida, aunque se mantenga inocuo.

Como esta misma definición ya sugiere, la alteración de los alimentos no afecta necesariamente a su seguridad, en el sentido de aparición de toxicidad. No obstante, especialmente si la alteración es intensa, se pueden formar compuestos tóxicos. 
Seguridad y toxicidad de los alimentos: un debate actual

Las modificaciones que pueden sufrir los alimentos a lo largo de la cadena alimentaria son de naturaleza química, física o microbiológica. Las consecuencias pueden ser de tipo higiénico-sanitario, sensoriales o nutricionales. Es importante destacar que las repercusiones de algunas modificaciones pueden ser favorables o desfavorables en función de la intensidad y del alimento de que se trate. Una de las causas más importantes de alteración de los alimentos es el desarrollo de microorganismos, hongos, levaduras o bacterias, a lo que hay que añadir los efectos de la infestación por insectos y roedores. En general, en la alteración de los alimentos de origen animal predominan las bacterias, mientras que en los de origen vegetal lo hacen los hongos y las levaduras. Esto explica que suelan ser más nocivos los alimentos de origen animal deteriorados que los de origen vegetal.

Como productos tóxicos resultantes de la alteración química y bioquímica de los alimentos podemos citar algunos de los formados en las llamadas reacciones de pardeamiento u oscurecimiento, si se dan con demasiada intensidad, o como consecuencia de la oxidación y consiguiente enranciamiento de las grasas.

\section{Adulteración y fraude de los alimentos}

Según el Código Alimentario Español tendrá la consideración de adulterado todo alimento al que se haya adicionado o sustraído cualquier sustancia para variar su composición, peso o volumen, con fines fraudulentos o para encubrir o corregir cualquier defecto debido a ser de inferior calidad o a tener ésta alterada. Este mismo código considera falsificado todo alimento en el que se haga concurrir alguna de las siguientes circunstancias: a) Que haya sido preparado o rotulado para simular otro conocido, b) Que su composición real no corresponda a la declarada y comercialmente anunciada, y c) Cualquier otra capaz de inducir error al consumidor.

Los motivos de la adulteración o la falsificación de alimentos son económicos y, en la mayoría de los casos, afectan a la honradez en las transacciones comerciales y no a la salud del consumidor. No obstante, ha habido casos en que la adulteración de un alimento ha dado lugar a consecuencias que han afectado la salud de los consumidores. Baste recordar el síndrome del aceite tóxico que afectó a España en 1981, sobre el que todavía subsisten dudas acerca de sus causas, aunque todo parece apuntar a que se debió a que se hizo pasar aceite de colza desnaturalizado para usos industriales por aceite de cocina. Las 
manipulaciones químicas que se llevaron a cabo en el aceite de colza (adición de anilina), unidas a los tratamientos de refinado posteriores, adecuados para el aceite para consumo humano pero no para un aceite de colza desnaturalizado, originaron los compuestos tóxicos responsables de una gravísima intoxicación de origen alimentario.

Entre los fraudes que más o menos siguen dándose y que pueden afectar a la seguridad de los alimentos podemos citar: adición de persulfatos a las harinas de trigo, adición de ácido bórico a los crustáceos (gambas) y presencia de cloropicrinas en vinos. Sin embargo, hemos de añadir que no son muy frecuentes y que, en general, no revisten un peligro realmente importante.

\section{Casos actuales que cuestionan la seguridad de los alimentos}

El tema de la seguridad alimentaria se ha venido manteniendo de actualidad en los últimos años por diversos casos, que no vamos a discutir con detalle sino que simplemente se comentarán para valorar su significación y hasta donde constituyen el reflejo de una situación. En este sentido pueden resultar ilustrativos los que se exponen a continuación:

\section{1) La crisis de las vacas locas}

La encefalopatía espongiforme bovina (EEB) se describió por primera vez en el Reino Unido en 1985, aunque se han presentado casos también en otros países. Parece ser que esta enfermedad neurodegenerativa ha sido transmitida a los animales bovinos a través de piensos elaborados con harinas de animales afectados por scrapie, una enfermedad del mismo tipo en ovejas y cabras ya descrita en el siglo XVIII. En la elaboración de estos piensos se había modificado la tecnología para reducir costos, disminuyendo la temperatura de tratamiento y variando el uso de disolventes. La enfermedad más conocida en el ser humano del mismo tipo es la llamada enfermedad de Creutzfeld-Jakob (ECJ). En un principio se pensaba que había una barrera entre especies y que la enfermedad no podía ser transmitida de una especie animal a otra y tampoco de un animal al hombre. Sin embargo la posibilidad de que la ECJ esté producida por un agente transmisible (una proteína designada como prión) sembró la alarma y la consiguiente caída en picado del mercado de la carne del Reino Unido. Ante la gravedad 
del riesgo deben adoptarse y se adoptaron medidas, como la inmovilización de productos derivados de los animales afectados o presuntamente afectados y el sacrificio de un gran número de ellos. Aún subsisten interrogantes y son necesarios más estudios epidemiológicos y sobre las vías de transmisión de los priones entre especies. En cuanto a las precauciones obligadas deben impedirse los contactos del agente productor de la EEB con animales y personas y desarrollar sistemas de vigilancia epidemiológica sobre estas enfermedades neurodegenerativas en seres humanos y animales. La gestión de la crisis por parte de las autoridades del Reino Unido y de la Unión Europea fue, entre otras cosas, lenta, poniendo de manifiesto, y ello es importante, que un mismo departamento de la administración de la Gran Bretaña se ocupaba de los intereses de los productores de animales y de los consumidores. Sin ser demasiado desconfiados, parece bastante evidente que la lógica visión "productivista» del ganadero no será la misma que la del consumidor. El control de los alimentos siempre debe ser llevado a cabo con una visión sanitaria y es necesario que este control sea independiente de la producción, por lo menos en lo que respecta a las decisiones finales a tomar, lo que no significa valorar insuficientemente las repercusiones económicas de un decomiso de reses

\section{2) El escándalo de los pollos belgas}

En enero de 1999 un avicultor belga observó trastornos y muertes en muchos de sus pollos. La causa era una contaminación espectacular del pienso por dioxinas, conjunto de compuestos químicos policlorados que incluyen las dioxinas propiamente dichas y los furanos. Se trata de substancias muy estables y por lo tanto con gran capacidad de permanencia en el medio ambiente, que se forman siempre que hay una combustión de materia orgánica en presencia de cloro (incineradoras de residuos domésticos e industriales y otras actividades industriales) y como subproducto de la fabricación de muchas substancias químicas como herbicidas, plaguicidas y otros productos. El riesgo radica en su estabilidad en el organismo y en la cronicidad de sus efectos. En el caso de los pollos belgas se trataba de una contaminación accidental, cuyo origen era un lote de grasas animales a las que se añadieron aceites de fritura recuperados indebidamente y que, a su vez, estaban contaminados con aceite de motores, siendo este último el responsable de la toxicidad, ya que la fritura no puede dar lugar a la formación de dioxinas. Se trata de un hecho grave pero excepcional, que también 
fue tratado con lentitud por las autoridades responsables, belgas en este caso. La psicosis fue extraordinaria y baste citar como ejemplo anecdótico pero ilustrativo el que en nuestro país se llegara a inmovilizar unas galletas simplemente porque la marca que figura en su etiquetado es «Belga». El problema fue serio, ya que un hombre de 70 kilos que comiera 150 gramos de pollo contaminado sobrepasaría el límite máximo permitido de dioxinas en 10 veces, lo cual es mucho en un día. No obstante, cabe recordar que sobrepasar las dosis máximas fijadas representa un riesgo cuando esta ingestión es reiterada, pero puede ser insignificante si se trata de un hecho aislado. Por lo tanto, el hecho es grave pero seguramente, aun cuando no disponemos de suficientes datos, no tanto como pareció percibirse por parte de los consumidores.

\section{3) Las carnes con hormonas}

Entre los productos que se utilizan fraudulentamente en producción animal se encuentran diversos compuestos de los que podemos considerar como paradigmático el clembuterol, substancia de carácter adrenérgico. Ya en 1990 se produjo en España una intoxicación alimentaria debida al consumo de hígado de animales bovinos (135 casos) cuya causa fue el uso ilícito de un fármaco beta-agonista, el clembuterol. Este fármaco se usa, a veces asociado a tireostáticos, para obtener carnes más magras e incrementar el peso de los animales con una notable reducción del costo de producción. El criterio de la Unión Europea en este ámbito es restrictivo, porque subsisten residuos, preferentemente en el hígado, que pueden afectar al consumidor, en especial si sufre ciertas patologías cardiovasculares. Aquí cabe hacer consideraciones de todo tipo, sobre todo económicas. Probablemente una política más realista de precios, con una adecuada información al consumidor de que la carne de calidad adecuadamente obtenida tiene un costo, sería un buen camino.

\section{4) Los antibióticos como residuos en productos cárnicos}

'Se ha afirmado que una de las posibles causas del desarrollo de resistencia a los antibióticos por parte de bacterias, lo cual constituye un problema sanitario importante, es el uso de antibacterianos en la producción animal, con fines profilácticos y quimioterápicos y también como promotores del crecimiento. Asimismo se ha atribuido a este uso 


\section{Seguridad y toxicidad de los alimentos: un debate actual}

de antibacterianos la aparición de ciertas alergias. Las actuales técnicas de producción, con animales hacinados y muy uniformes en sus características, los hacen especialmente vulnerables a plagas masivas, que es lo se trata de evitar y/o compensar con estas técnicas. Debe quedar claro que el problema podría darse cuando haya un mal uso de estos fármacos, pero no se estima que sea así si se aplican en dosis subterapéuticas para facilitar la crianza de las aves. Es de señalar que se preconiza que los antibióticos que se usen con estas finalidades sean distintos de los utilizados en medicina humana. En definitiva, no está probado realmente que el fracaso de ciertos antibióticos en medicina humana sea atribuible a una resistencia adquirida a causa de la ingestión de productos de origen animal contaminados con agentes antibacterianos, y no se deba a otras causas. Recordemos que el abuso de antibióticos en España en terapéuticas inadecuadas (automedicación en muchas ocasiones) es elevado. El desarrollo de nuevas técnicas de alimentación y cría más acordes con la salud y bienestar de los animales reduciría sin duda estos eventuales riesgos, aunque quizás aumentaría los costos.

\section{5) La Coca-Cola contaminada}

En junio de 1999 se retiraron en Bélgica y Francia los productos de Coca-Cola después que unos cuarenta niños de una escuela fueran hospitalizados con trastornos que no revistieron gravedad. Se planteó como causa la presencia de sulfuro de hidrógeno en los productos afectados y también se aventuraron otras hipótesis (uso de dióxido de carbono impurificado e incluso productos del tratamiento de los embalajes). Automáticamente se registraron muchos casos parecidos, en localizaciones geográficas diversas y con síntomas heterogéneos. No se ha encontrado el tóxico responsable. Finalmente, aunque subsisten ciertos interrogantes, parece que se ha tratado simplemente, como decía el periódico francés Le Figaro, el 30 de diciembre de 1999, de una «enfermedad de masas determinada sociológicamente».

Podríamos citar otros casos como la presencia de Listeria en productos cárnicos franceses o de Escherichia coli 0157:H7 en Japón, Estados Unidos o el Reino Unido.

Es importante destacar que el sector implicado en la mayoría de estos casos en los que realmente se ha dado un problema es el de la producción primaria de alimentos y no el industrial. Ello pone de manifiesto, como han señalado Focant y de Pauw (1999), que la ne- 
cesidad de control de la industria alimentaria no sólo debe ir «hacia abajo» sino también «hacia arriba». Es decir, ha de extenderse hacia el sector agrícola y ganadero productor de las materias primas. Esto es más difícil de llevar a la práctica, tanto por razones sociales y económicas como también por la naturaleza del sector. Podríamos decir que también en esto es difícil «poner puertas al campo».

Otro motivo de reflexión sería el que estos casos se han dado en países desarrollados. Ello es así, entre otras razones, porque si bien la industrialización de los alimentos permite, sin duda, disponer de técnicas de producción y control más seguras, la masificación de esta producción y de la distribución hacen que las consecuencias de cualquier incidente, aunque sea excepcional, adquiera grandes proporciones.

\section{El control alimentario como prevención}

Es una obviedad que la garantía de la seguridad alimentaria radica en un control completo y fiable de los alimentos a lo largo de toda la cadena de producción y distribución. En este sentido ha cambiado el concepto y la ejecución de este control. La idea clásica se basaba en el análisis de los productos acabados en la industria. Como es lógico, esto sólo servía para aceptar o descartar los productos. En la actualidad, el criterio que se ha impuesto en todos los niveles es el preventivo. Podemos ilustrarlo, por ejemplo, indicando que el control para asegurar la calidad de una leche de vaca empieza en las granjas en las que se encuentran los animales productores. Es fácil entender que si ya a este nivel se hacen las cosas bien, la leche llegará a la planta de tratamiento en buenas condiciones, y los consiguientes tratamientos de higienización podrán ser más suaves para conseguir y garantizar una leche no sólo segura desde el punto de vista microbiológico, sino que también mantenga al máximo sus características sensoriales y nutricionales. El desarrollo de estos criterios ha llevado a la implantación cada vez más generalizada del Sistema llamado de Análisis de Riesgos e Identificación y Control de Puntos Críticos (ARICPC), en virtud del cual se lleva a cabo un estudio de todas las etapas que comprende el proceso de obtención y tratamiento de un alimento y se evalúan cuáles son las etapas o puntos más delicados de toda esta secuencia, para dedicar a ellos una atención preferente. Siguiendo con el ejemplo de la leche, el control riguroso de la temperatura y del tiempo de tratamiento térmico correspondiente es fundamental. Si la materia prima ya llegó en buenas condiciones y este 
proceso ha sido correcto, el análisis final del producto elaborado será una comprobación necesaria pero no la operación clave o crítica para garantizar la calidad de la leche. Es decir, si limitamos los aportes microbianos y su multiplicación, no deberemos forzar los tratamientos para destruir microorganismos con la consiguiente merma en la calidad del producto, la cual conlleva mucho más que cumplir con las mínimas condiciones higiénico-sanitarias. Por lo tanto, el control no se reduce a los meros análisis concretos, que son una herramienta pero no la esencia del proceso. Por esto hoy se puede decir que la calidad de un producto no se analiza, sino que se gestiona en virtud de unas «Buenas Prácticas de Fabricación», que a partir de unas pautas bien determinadas apelan a la responsabilidad de todos los agentes que intervienen en el proceso y no sólo de los que al final controlan el producto. Es importante señalar que el sistema ARICPC para controlar los peligros y disminuir el riesgo real al máximo no sólo es aplicable para los eventuales contaminantes biológicos (especialmente microbiológicos), sino también a los de tipo químico. También debemos señalar que éste es un sistema implantado en la industria que no es fácil de extender a los productos frescos de origen estrictamente agrario, que por su especial naturaleza es donde hoy sigue habiendo más problemas como ya hemos señalado. En los aspectos estrictamente microbiológicos, la microbiología predictiva complementa el sistema ARICPC. La microbiología predictiva estudia el crecimiento de los microorganismos en los alimentos y los factores que los condicionan, para así poder predecir lo que eventualmente sucedería en el alimento durante su almacenamiento y distribución.

Dentro de toda esta perspectiva, vemos que la inspección y análisis de alimentos por parte de las Administraciones ya no es la única acción que garantiza la calidad de los alimentos, sino que la clave es la confianza en que la industria hace las cosas bien según los criterios expuestos, de cuyo cumplimiento la inspección simplemente dará cuenta o detectará sus fallos.

\section{El papel de las Administraciones Públicas}

La Declaración Universal de los derechos del Hombre reconoce que todas las personas tienen derecho a una alimentación suficiente y sana. Por ello las Administraciones públicas, y en Europa esto empieza por la Unión Europea, deben actuar para proteger la salud de los consumidores y velar por la seguridad de los alimentos. Como respuesta a 
todas estas inquietudes, la Comisión Europea ha puesto en marcha en todos los países de la UE una Campaña de Seguridad Alimentaria dirigida a los consumidores, poniendo en evidencia que el objetivo de alcanzar las máximas cotas de seguridad alimentaria requiere un ejercicio de responsabilidad compartida entre todos los eslabones que integran la cadena alimentaria: productores, transformadores y distribuidores, y también consumidores.

Debido, sobre todo, a los recientes y espectaculares casos de contaminación de alimentos en la Unión Europea, la Comisión Europea ha elaborado y presentado su «Libro Blanco sobre la Seguridad Alimentaria», en el cual se propone la creación de una Agencia Alimentaria Europea o Autoridad Alimentaria Europea, que se pretende que tenga funciones análogas a la Food and Drug Administration (Administración de Alimentos y Medicamentos) de los Estados Unidos. El objetivo último es aumentar los estándares de los productos alimenticios, aplicar principios estrictos de etiquetado y aumentar los controles y las inspecciones. Dicha Autoridad Alimentaria «independiente y transparente» tendrá como principal objetivo la confirmación y comunicación de los riesgos en el ámbito alimentario, aunque la gestión de los riesgos (incluyendo la legislación y el control) continuará siendo competencia de las instituciones comunitarias y de los Estados. Esta Autoridad, cuya sede está pendiente de decisión, deberá colaborar estrechamente con los organismos científicos de los estados miembros y con la Comisión Europea. También ha habido respuestas a estas contaminaciones de alimentos de las administraciones estatales y de las comunidades autónomas en España. Todas estas reacciones son lógicas, pero no podemos por menos que temer que por querer evidenciar capacidad de reacción no se tomen medidas apresuradas o excesivas. En España, después del síndrome del aceite tóxico se dio una gran aceleración legislativa, que alguien calificó de "legislatorrea», que no siempre condujo a resultados realmente positivos.

\section{Conclusiones y perspectivas}

La comunidad científica debería transmitir con claridad, y recabar la confianza del consumidor al respecto, que como indica Golberg todo lo que un toxicólogo puede conseguir es valorar el grado en el que unos determinados niveles de contaminantes, en unos determinados alimentos, consumidos en cantidades definidas y con una frecuencia también conocida, añaden algún riesgo a los diversos tipos de riesgos 
potenciales ya preexistentes que puede haber en una dieta. Como ya se ha indicado, por lo menos de momento, la dieta absolutamente inocua para cualquier persona o colectivo no existe, aunque debe ser un permanente objetivo a alcanzar.

Hay que exigir datos científicamente consistentes, criterios de evaluación juiciosos $\mathrm{y}$, por encima de todo, libertad frente a cualquier tipo de presión ajena. De esta manera seguiremos en el buen camino de irnos acercando «asintóticamente» a la alimentación segura.

Nos parece que puede ser una muy adecuada reflexión final la que hacían Egeland y Middaugh en diciembre de 1997 en Science. Con muy buen criterio estos autores critican la tendencia a reducir el consumo de pescado por su eventual contenido en metilmercurio y otros derivados orgánicos del mercurio, ya que ello lleva a perder los efectos positivos del consumo de pescado. En efecto, los criterios restrictivos que pretenden aumentar la seguridad olvidan, en este caso, los beneficios derivados del consumo de pescado, y en especial el graso, que contribuye a disminuir el riesgo de trastornos cardiovasculares y tiene otros efectos beneficiosos, por su contenido elevado en proteínas, bajo en grasas saturadas, ser vehículo de ácidos grasos poliinsaturados omega-3 y de antioxidantes como selenio y vitamina E. Además, estos mismos componentes del pescado, en especial el selenio y la vitamina $\mathrm{E}$, pueden incluso proteger frente a los efectos negativos de bajas dosis de metilmercurio. Si consideramos debidamente el valor en conjunto de los alimentos, que incluye sus características nutricionales y no sólo de seguridad, no caeremos en el error de por una precaución excesiva frente a un contaminante prescindir de otras propiedades cuyo impacto positivo es superior al eventual impacto negativo de una contaminación. Por lo tanto, una correcta aplicación de la evaluación riesgo/beneficio nos lleva a la conclusión de que es mejor ingerir pescado aún asumiendo una cierta contaminación con metil mercurio que suprimirlo de la dieta, a no ser que se tratara de niveles significativamente elevados. Ni que decir tiene que esto no debe significar que se acepta «resignadamente» la contaminación por organomercuriales.

Como en cualquier actividad humana, y parafraseando a Kierkegaard «vivir es elegir». Los científicos y tecnólogos, en contacto con los clínicos y los productores de alimentos, deben seguir investigando sobre la seguridad de los alimentos y aportar nuevos datos, certezas o dudas, que deben ser divulgados con rigor y claridad a la población en general, la cual, como hicieron los diabéticos norteamericanos a que antes aludimos, en uso de su libertad elegirá. En definitiva, aunque 
seguimos teniendo problemas, nunca el conjunto de nuestros alimentos fue tan seguro como en la actualidad.

\section{Bibliografía}

Centrich-Sureda, M., 1999: Adulteraciones alimentarias. Contaminación de alimentos. En: Hernández-Rodríguez, M. y Sastre-Gallego, A.(Directores) Tratado de Nutrición: 475-489. Díaz de Santos, Madrid.

Concellón-Martínez, A, 1995: Salud Pública y residuos de medicamentos veterinarios en los alimentos. Alimentaria, Septiembre: 87-100.

Consorcio para la SEguridad alimentaria, 1999: Mitos y Realidades de la Seguridad Alimentaria: 59 pp., Madrid.

Daniels, R.W., 1998: Home Food Safety. Food Technology, 52 (2): 54-56.

Domínguez, A., Mata, E., Salleras, L., 1998: Los priones y las enfermedades neurovegetativas transmisibles. Medicina clinica, 110 (19): 751-757.

Egeland, G.M., Middaugh, J.P., 1997: Balancing Fish Consumption Benefits with Mercury Exposure. Science, 278: 1904-1905.

EUFIC (Consejo Europeo de Información sobre Alimentación), 1998: ¿Es fiable nuestra alimentación? Foodtoday, 9: 1-4.

FischleR, C., 1998: Raison et déraison dans la perception des risques alimentaires. Cah. Nutr. Diét., 33 (5): 297-300.

Focant, J.-F., DE Pauw, E., 1999: Las dioxinas. Mundo Científico, 206: 84-87.

Golberg, L., 1971: Trace Chemical Contaminants in Food: Potential for Harm. Food Cosmet. Toxicol, 9: 65-80.

Herrera-Marteache, A., Conchello-Moreno, P., 1999: La cadena alimentaria como riesgo para la salud pública. Contaminación y alteración alimentaria. En: HernándezRodrfguez, M. y Sastre-Gallego, A. (Directores): Tratado de Nutrición: 503-541. Díaz de Santos, Madrid.

ILSI EUPOPE NOVEL FOOD TASK FORCE, 1996: The Safety Assessment of Novel Foods. Food and Chemical Toxicology, 34: 931-940.

Iterg, 1999: Dioxine. Ce qu'il faut savoir. Bull. Institut Français pour la Nutrition, 70: $54-60$.

KEEnE, WE, 1999: Lessons From Investigations of Foodborne Disease Outbreaks. JAMA, 281 (19): 1845-1847.

MARINÉ-FonT, A., VidAL-CAROU, M.C., 1996: Literatura y control alimentario. Alimentación, Nutrición y Salud, 3 (1): 18-21.

Mariné-Font, A., 1981: Toxicidad natural en los alimentos. Alimentaria, 120: 33-39.

Mariné-Font, A., Vidal-Carou, M.C., Hernández-Jover, T., 1999: Aditivos alimentarios. En: Hernández-Rodriguez, M. y Sastre-Gallego, A. (Directores): Tratado de Nutrición: 465-474. Díaz de Santos, Madrid.

Martín, R., Hernández, P.E., Sanz, B., 1992: Residuos de tratamientos veterinarios y salud pública. Revista Española de Ciencia y Tecnología de Alimentos, 32 (5): 461-480.

Martínez-NaVARro J.F., 1991: Food poisoning related to consumption of illicit beta-agonist in liver. The Lancet, 336, 1311. 


\section{Seguridad y toxicidad de los alimentos: un debate actual}

MauRICE, J., 1994: The rise and rise of food poisoning. New Scientist, 1956: 28-33.

Mónico-Pifarré, A., Mariné-Font, A., Xirau-Vayreda, M., 1986: Impurezas de origen no natural en alimentos. Alimentaria, Julio-Agosto: 21-32.

Moreno, B., 1995: Posibilidades actuales y futuras para el control y la prevención, por parte de las industrias, de las enfermedades transmitidas por los alimentos. Alimentaria, Junio: 41-52.

Moreno-Garcí, B., García-Armesto, M.R., López, T.M., 2000: Riesgos sanitarios de la contaminación de alimentos. Alimentaria, Enero-Febrero: 19-28.

Notermans, S., Borgdorff, M., 1997: A Global Perspective of Foodborne Disease. J. Food Protection, 60 (11): 1395-1399.

Patterson, R.E., Kristal, A.R., Lynch, J.C., White, E., 1995: Diet-Cancer Related Beliefs, Knowledge, Norms, and Their Relationship to Helthful Diets. J. Nutrition Education, 27 (2): 86-92.

Piqueras, M., 2000: Rachel Louise Carson, el poder de una idea. Mujeres y Salud, 5: 12-14.

Repetto, M., Cameán, A., 1996: Venenos naturales en los alimentos. Alimentación, $\mathrm{Nu}$ trición y Salud, 3 (4): 78-86.

SCANLAN, R.A., 1995: Volatile nitrosamines in foods - an update. En Charalambous, G. (Ed): Food Flavors: Generation, Analysis and Process Influence: 685-704. Elsevier.

Sugimura, T., 1995: History, present and future, of heterocyclic amines, cooked food mutagens. En Adamson et al. Heterocyclic Amines in Cooked Foods: Possible Human Carcinogens: 214-231. Princeton Sci. Pub., Princeton, New Jersey.

Vidal-Carou, M.C-., Izquierdo-Pulido, M., Veciana-Nogués, T., 1999: Estabilidad y métodos de conservación de los alimentos. En: Hernández-Rodríguez, M. y Sastre-Gallego, A. (Directores): Tratado de Nutrición: 451-464. Díaz de Santos, Madrid.

Villalbí, J.R., Llebarta, X., Armengol, R., Plasencia, A., Armengou, J.M., 1998: La higiene y seguridad de los alimentos dentro del sistema de información sanitaria. Rev. Esp. Nutr. Comunitaria 4 (4):332-337.

Winter, C.K., Francis, F.J., 1997: Assessing, Managing, and Comunicating Chemical Food Risks. Food Technology, 51 (5): 85-92.

WiтTE, W., 1998. Medical Consequences of Antibiotic Use in Agriculture. Science, 279: 996-997. 